\title{
Benign asbestos pleural effusion: diagnosis and course
}

\author{
BWS ROBINSON, AW MUSK \\ From the Department of Respiratory Medicine, The Queen Elizabeth Medical Centre, Nedlands, \\ Western Australia
}

ABSTRACT We have reviewed 22 patients with benign asbestos pleural effusion seen over a 17-year period. The mean duration of exposure to asbestos was 5.5 years and the mean interval between exposure and presentation was 16.3 years. In five the effusion was asymptomatic. Fever was uncommon but in 15 of 21 patients the ESR was elevated. Leucocytosis was noted in seven of 20 patients. Autoantibodies were rarely detected. The pleural fluid was usually blood-stained and the volume aspirated was rarely larger than $500 \mathrm{ml}$. Pleural biopsies revealed established pleural fibrosis and/or inflammatory infiltration with fibrinous exudate and mesothelial and fibroblastic proliferation. A positive mantoux test was noted in eight of 12 patients but there was no other evidence of tuberculosis. The mean duration to spontaneous resolution of the effusion was $4 \cdot 3$ months. During a follow-up period of $28 \cdot 1$ years from initial exposure to asbestos (mean $22 \cdot 8$ years) and up to $17 \cdot 2$ years from initial presentation with a pleural effusion (mean 6.3 years) seven patients had a single recurrence and only one patient had multiple pleural effusions. Only three patients experienced persistent pleural pain. It was not possible to predict the likelihood of recurrence of an effusion or the persistence of pleural pain from the data at presentation. No patient subsequently developed mesothelioma or other neoplasm.

Since the initial description of benign asbestos pleural effusion in $1964^{1}$ the clinical and laboratory features of about 40 cases have been reported. ${ }^{2-9}$ The generally accepted diagnostic criteria are (i) previous asbestos exposure (ii) presence of pleural effusion without demonstrable alternative cause, and (iii) spontaneous resolution or non-recurrence after aspiration. ${ }^{2}$ The condition has only been recognised over the past 16 years and so far there has been no detailed study undertaken to document the range of presenting features and the outlook for persistence of pleural pain, occurrence of further effusions and the development of malignant pleural mesothelioma. We thus undertook this retrospective study of 22 such patients.

\section{Methods}

Twenty-two patients of the Department of Respiratory Medicine seen over the past 17 years fulfilled the

Address for reprint requests: Dr BWS Robinson, Department of Respiratory Medicine, The Queen Elizabeth II Medical Centre, Nedlands 6009, Western Australia. above diagnostic criteria. Six of these cases have been mentioned briefly in previous communications. ${ }^{810}$ Pleural effusions were confirmed by aspiration of fluid or radiological resolution.

Data relating to presentation and follow-up were obtained from the hospital case records and by telephoning the patient himself and/or his general practitioner. Pleuritic pain was taken as localised chest pain, sharp in nature and worse on inspiration and/or coughing in the absence of local musculoskeletal disease. ${ }^{11}$ Pleural thickening was recorded if the width of the pleural shadow on the chest radiograph was increased, irrespective of the extent of the thickening (pleural effusion having been excluded). Pleural plaques were defined as localised areas of pleural thickening covering less than four interspaces excluding the lung apices and costophrenic angles with or without associated calcification. ${ }^{12}$ Radiological pneumonconiosis was recorded if diffuse nodular or reticulonodular shadowing was found on chest radiograph. ${ }^{12}$

Rheumatoid factor was assessed using the Rose and Ball (Difco), Latex (Hyland) or Rheumaton (Denver Lab) methods, positive titres being taken as 
1 in 64, 1 in 16, and 1 in 8 respectively.

Mantoux testing was performed with human Purified Protein Derivative (Commonwealth Serum Laboratories) and a result was considered positive if the diameter of induration was greater than $10 \mathrm{~mm}$ using 10 tuberculin units. ${ }^{13}$ Forced expiratory volume in one second $\left(\mathrm{FEV}_{1}\right)$ and vital capacity $(\mathrm{VC})$ were measured spirometrically. Total lung capacity (TLC) was measured plethysmographically. Transfer factor (TL) was measured by the single breath carbon monoxide technique. ${ }^{14}$ Values were normalised for haemoglobin and corrected for expired $\mathrm{CO}_{2}$. Effective alveolar volume $\left(\mathrm{VA}_{\mathrm{A}}\right)$ was calculated from single breath helium dilution during breath-holding and corrected for dead space.

\section{Results}

All 22 patients were males. In no patient diagnosed as having a benign asbestos pleural effusion was the diagnosis subsequently altered. One patient had sero-negative arthritis of the rheumatoid type.

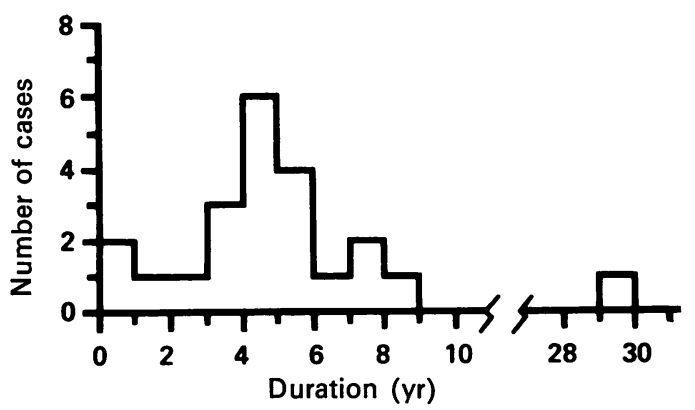

Fig 1 Duration of asbestos exposure before presentation.

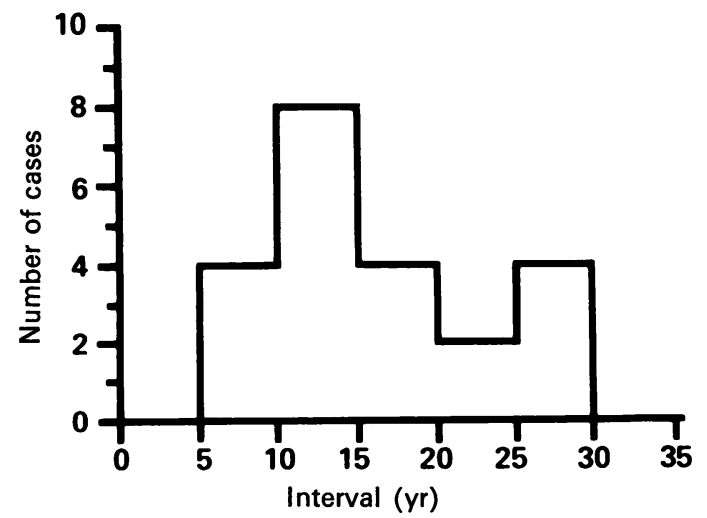

Fig 2 Interval between onset of exposure and presentation.
Of the 22 patients, 15 had been involved in the mining and/or milling of crocidolite ("blue asbestos") at Wittenoom Gorge in the North West of Western Australia, four were employed in the cutting of asbestos sheeting, one was exposed to asbestos spray while insulating railway coaches, one was a plumber exposed to asbestos lagging on pipes, and one man cleaned bags which previously had contained asbestos from Wittenoom Gorge and South Africa. The exposure of the patients was therefore mainly to crocidolite.

The duration of exposure to asbestos (fig 1) was as short as two weeks. Only one patient had been exposed for more than nine years (29 years) but his exposure (working with asbestos sheeting) was minimal and intermittent.

There was a broad range of time from initial exposure to the development of a pleural effusion (fig 2). Only four patients were still occupationally exposed to asbestos at the time of presentation. The Wittenoom mine was closed in 1966.

\section{CLINICAL FEATURES}

Thirteen patients presented in their fifties, most having started asbestos-related employment in their thirties. ${ }^{15}$

Pleuritic chest pain was the reason for presentation in 14 men. The mean duration of the pain was $9 \cdot 6$ weeks (range 2 to 26 weeks). Although fever was noted in three of 16 cases no one had a temperature above $37 \cdot 5^{\circ} \mathrm{C}$. Three men presented with dyspnoea while the other five were free of symptoms. Clinical findings are summarised in tables 1 and 2.

\section{LABORATORY RESULTS}

Peripheral blood leucocytosis was common but in no patient was the white cell count greater than $12.0 \times 10^{9} 1^{-1}$. In six of the seven men with leucocytosis there was an associated elevation of the erythrocyte sedimentation rate. Asbestos bodies were seen in the sputum of just under half of those patients in whom a sputum search was recorded.

In four men the volume of pleural fluid aspirated at initial presentation was $500 \mathrm{ml}$ or more (range 500 to $1500 \mathrm{ml}$ ). In 13 men the pleural fluid was frankly bloodstained. There was no correlation between the severity of the presenting features as adjudged by leucocytosis, fever, or elevation of the ESR and the presence of blood-staining of the pleural effusion. Of the five samples of pleural fluid in which the leucocytes were eosinophils, four were also blood-stained. The protein content of the pleural fluid was greater than $30 \mathrm{~g} / \mathrm{l}$ in all seven in which it was recorded. No patient had evidence of bacterial infection of the pleural space.

Acid-fast bacilli were not seen or cultured from 
Table 1 Clinical features at presentation

\begin{tabular}{lccc}
\hline Clinical feature & Present & Absent & Not recorded \\
\hline Pleuritic pain & 14 & 8 & 0 \\
Current or ex-smoker & 20 & 2 & 0 \\
Clubbing & 0 & 22 & 0 \\
Crackles & 4 & 18 & 0 \\
Fever (> 37 $\left.{ }^{\circ} \mathrm{C}\right)$ & 3 & 13 & 6 \\
Pleural rub & 5 & 17 & 0 \\
\hline
\end{tabular}

Table 2 Laboratory abnormalities at initial presentation

\begin{tabular}{|c|c|c|c|}
\hline Laboratory abnormality & Present & Absent & Not recorded \\
\hline $\begin{array}{l}\text { Elevated erythrocyte sedimentation } \\
\text { rate ( }>15 \mathrm{~mm} / \text { hour-Westergren) }\end{array}$ & 15 & 6 & 1 \\
\hline $\begin{aligned}>15 & <50 \\
& \geqslant 50\end{aligned}$ & $\begin{array}{r}10 \\
5\end{array}$ & & \\
\hline $\begin{array}{l}\text { Peripheral blood leucocytosis } \\
\left(>9.0 \times 10^{\circ} / 1\right)\end{array}$ & 7 & 13 & 2 \\
\hline Asbestos bodies in sputum & 7 & 8 & 7 \\
\hline Rheumatoid factor positive & 0 & 10 & 12 \\
\hline Antinuslear factor positive & 2 & 10 & 10 \\
\hline Mantoux positive & 8 & 4 & 10 \\
\hline Pleural fluid aspirated & 20 & 2 & 0 \\
\hline Blood-stained & 13 & 4 & 3 \\
\hline Eosinophils $>50 \%$ & 5 & 10 & 5 \\
\hline Lymphocytes $>50 \%$ & 8 & 7 & 5 \\
\hline Neutrophils $>50 \%$ & 2 & 13 & 5 \\
\hline
\end{tabular}

pleural fluid, sputum, or gastric washings and in no patient did pleural biopsy show evidence of tuberculous granulomata. No patient had anti-tuberculous therapy, and none subsequently developed tuberculosis.

All three men with reduced $\mathrm{FEV}_{1} / \mathrm{VC}$ ratios were smokers. Changes from predicted values of the pulmonary function tests could be attributed to the volume of pleural fluid in the chest. There was no overall functional evidence of airway narrowing or pulmonary fibrosis.

Of 14 adequate pleural biopsies, seven showed fibrinous exudate with inflammatory cells and early fibroblastic proliferation; the other seven showed established pleural fibrosis. In no pleural biopsy were asbestos bodies seen. Lung biopsies were performed in two patients only and both revealed pulmonary fibrosis with asbestos bodies. Only one of these had radiological asbestosis.

\section{COURSE OF THE DISEASE}

Two patients died of unrelated causes soon after diagnosis (one of a subarachnoid haemorrhage, the other of ischaemic heart disease) but neither had evidence of mesothelioma at the time of death. The remaining 19 cases have been followed up from three months to 17.2 years (mean 6.3 years) after their initial diagnosis and from nine to 34 years (mean 22.8 years) after their initial exposure to

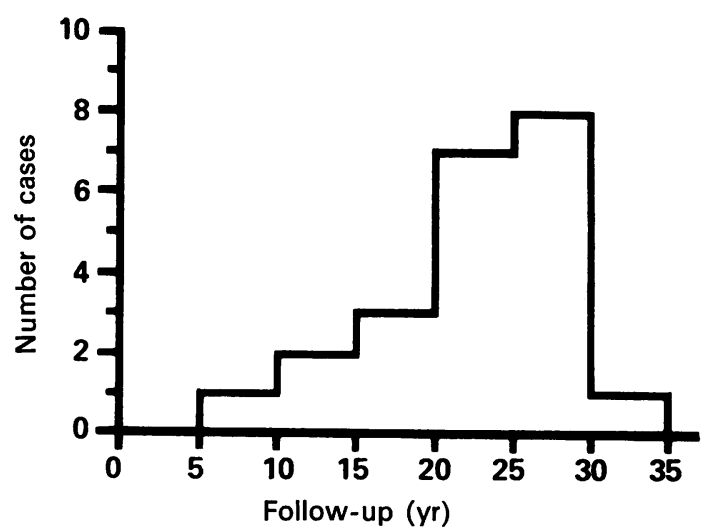

Fig 3 Follow-up since initial exposure to asbestos.

asbestos (fig 3). Nine have been followed up for more than 10 years after their initial presentation.

In four patients the effusion did not recollect after initial aspiration. One patient had a surgical pleurectomy during his initial admission and has remained free of recurrence. In one it was not possible to assess the duration of the initial effusion. In the remaining 16 the mean resolution time was $4 \cdot 3$ months (range one to 12 months). In no patient did the effusion persist for longer than 12 months after diagnosis.

Eight patients had more than one pleural effusion. Six had one contralateral occurrence only, one case had one ipsilateral recurrence only, and one case had one contralateral occurrence and two ipsilateral recurrences. The interval between initial effusion and contralateral effusion varied from 0 (synchronous) to $4 \cdot 8$ years (mean $17 \cdot 1$ months). It was not possible to define features at presentation that would predict the occurrence of further effusions or the persistence of pleuritic pain.

None of the patients has developed evidence of a pleural or peritoneal mesothelioma nor of any other neoplasm during follow-up. Three patients have continued to experience persistent pleuritic pain after resolution of the initial effusion. They have been followed up for three, four, and eight years respectively after their initial presentation. Only one of these has subsequently developed a second effusion.

None of the 10 with clear lungs radiologically and $c$ clinically at presentation has subsequently developed radiographic evidence of asbestosis during a mean follow-up time of four years (range of 0.5 to 11.1 years). Pleural thickening was evident during the initial presentation in 16 patients and became evident in a further two during follow-up. No patient developed massive pleural thickening requiring 
pleurectomy and no patient developed radiological evidence of pleural plaque formation.

\section{Discussion}

The size of this series reflects both the geographical proximity of this hospital to an asbestos-mining area and the local awareness among physicians and patients of asbestos-related diseases. The tendency of patients to present in the fifth decade of life reflects the age at which they started working with asbestos and the latent period between initial exposure and presentation. The spectrum of presentations of benign asbestos pleural effusion lies between that of an unwell patient with a fever, leucocytosis, elevated ESR, pleuritic pain, and blood-stained pleural effusion through to that of an otherwise well young man with his effusion diagnosed incidently on routine chest radiography.

Most of the presenting clinical and laboratory features are consistent with previous reports. Preceding pleuritic pain ( $64 \%$ of cases) was more frequently found and clubbing $(10 \%)$ and crackles $(18 \%)$ were less frequently found than in Gaensler's series $^{3}$ where $33 \%$ presented with pleuritic pain, $33 \%$ were clubbed and $42 \%$ had crackles at presentation. Although in five patients eosinophils represented the majority of white cells found in the pleural fluid, in four there was associated blood-staining which is probably sufficient to explain the phenomenon. ${ }^{13}$ This feature has been noted previously. ${ }^{5}$

Reduction in TLC, VC and TL at presentation were not unexpected findings in view of the presence of pleural fluid. A recent study has suggested that a normal value for $T_{L} / V_{A}$ in patients with asbestosrelated pleural disease indicates that very little parenchymal disease is present. ${ }^{16}$

The need to take an accurate occupational history in patients with pleural effusion is emphasised, particularly as the exposure may be minimal, asbestos bodies are rarely found in the pleural tissue $^{19}$ and asbestos exposure is often unsuspected by clinicians. ${ }^{20}$ Other causes of the effusion likewise require careful exclusion. It is likely that patients with this condition have in the past been labelled as having tuberculous effusions, a diagnosis that would have been reinforced by a positive mantoux test and an apparent resolution of the effusion over the ensuing few months on anti-tuberculous therapy. One patient had initially been labelled as "presumed viral pleurisy with effusion" despite unhelpful viral titres. Systemic features at presentation had in several cases in this series suggested a recent viral infection. Sequential viral studies were performed in only two cases. Whether asbestos-related pleural disease predisposed patients with viral infections to the develop- ment of pleural effusions could not be determined from the data available. It is possible that intercurrent viral infection brings the patient to medical attention.

Since effusions were often quite small, often associated with few symptoms and sometimes found incidentally it is likely that many go undetected. It has been suggested that pleurisy with a small or large effusion may be a frequent occurrence during the development of asbestos-induced pleural fibrosis.

The mean resolution time of four months for the initial effusion is consistent with the findings in previous reports. $^{2-9}$ Recurrences however were considerably less common in this series ( $36 \%$ of cases) than in others and only one patient had more than two effusions. Further effusion is most likely within three years of initial presentation, and it is not possible to predict the likelihood of further effusion or persistence of pleuritic pain in any individual patient.

Although none of these cases has been shown to have developed mesothelioma or bronchogenic carcinoma their risks are increased over those of the general population because of their asbestos exposure and smoking habits. ${ }^{2} 39101718$ Benign asbestos pleural effusion appears to occur fairly early in the natural history of asbestos-related lung disease and follow-up of some of the above cases has not yet reached the 20-40 year interval after initial exposure, the interval of peak mesothelioma incidence. ${ }^{2} 141718$ The absence of radiological evidence of calcified pleural plaque formation probably also reflects the early occurrence of benign pleural effusion in the natural history of asbestos-related lung disease, plaque formation being a late feature. ${ }^{8}$

The results of this study of 22 patients with benign asbestos pleural effusion emphasise the heterogeneity of the clinical features at presentation and indicate that in these cases further effusions and persistent pleural pain are uncommon and malignant change not yet documented.

We would like to thank Dr J Elder, Dr AR Adams, Dr HR Elphick, and Dr A Tribe for their permission to publish details of cases under their care, Dr KE Finucane, Dr J Baker, Dr J Pollard, and Mr S Woodward for their assistance and their editorial advice, and Mrs C Gellé for her help in the preparation of this manuscript.

\section{References}

${ }^{1}$ Eisenstadt HB. Asbestos pleurisy. Chest 1964;46:78-81.

2 Preger L. Asbestos-related disease. New York: Grune and Stratton, 1978.

${ }^{3}$ Gaensler EA, Kaplan AI. Asbestos pleural effusion. Ann Intern Med 1971 ;74:178-91. 
4 Chahinian PH, Hirsch A, Bignon J et al. Les pleurésies asbestosiques non tumorales. Rev Fr Mal Respir 1973; 1:5-39.

${ }^{5}$ Lémengager J, Rousselot P, LeBouffant L. Pleurésies asbestosiques non tumorales. Nouv Presse Med 1975;4: 1134.

B Nyiredy G. Benigne asbestpleuritis. Prax Pneumol 1975; 29:166-9.

' Sluis-Cremer GK, Webster I. Acute pleurisy in asbestos exposed persons. Environ Res 1972;5:380-92.

${ }^{8}$ Elder JL. A study of 16 cases of pleurisy with effusions in ex-miners from Wittenoom Gorge. Aust NZ J Med 1972; 2:328-9.

${ }^{9}$ Eisenstadt HB. Benign asbestos pleurisy. JAMA 1965;192: 419-21.

10 McNulty JC. Asbestos mining, Wittenoom, Western Australia, in Proceedings of the First Australian Pneumoconiosis Conference, Sydney. Sydney: New South Wales Joint Coal Board, 1968:447-66.

11 Lowell JR. Pleural effusions. Baltimore: University Park Press, 1977.

${ }^{12}$ Fletcher DE, Edge JR. The early radiological changes in pulmonary and pleural asbestosis. Clin Radiol 1970;21: 355-65.
${ }^{13}$ Crofton J, Douglas A. Respiratory diseases. Oxford: Blackwell, 1975:190.

14 Cotes JE. Lung function. Third edition. Oxford: Blackwell, 1977:243-5.

${ }^{15}$ Hobbs MST, Woodward S, Murphy B, Musk AW, Elder $\mathrm{JL}$. The incidence of pneumoconiosis, mesothelioma and other respiratory cancer in men engaged in mining and milling crocidolite in Western Australia. 1981; in press.

${ }^{16}$ Wright PH, Hanson A, Kreel L, Capel LH. Respiratory function changes after asbestos pleurisy. Thorax 1980; 35:31-6.

17 Kannerstein M, Churg J, McCaughey WTE. Asbestos and mesothelioma: a review. Pathology Annual 1978;13:80129.

${ }^{18}$ Becklake MR. Asbestos-related diseases of the lung and other organs; their epidemiology and implications for clinical practice. Am Rev Respir Dis 1976;114:187-227.

19 Eisenstadt HB. Pleural effusion in asbestosis. $N$ Engl J Med 1974;290:1025.

${ }^{20}$ Martischnig KM, Newell DJ, Barnsley WC, Cowan WK, Feinmann EL, Oliver E. Unsuspected exposure to asbestos and bronchogenic carcinoma. $\mathrm{Br}$ Med $J 1977$ 1:746-9. 